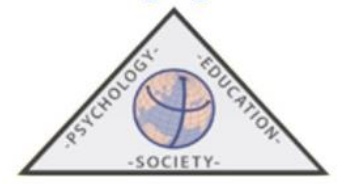

\title{
Medio penitenciario y la protección de los derechos humanos de las personas con discapacidad. Perspectiva desde las mujeres
}

\author{
Karen G. Añaños Bedriñana ${ }^{1}$, Francisco Sánchez González ${ }^{2}$ y Fernando Gil Cantero ${ }^{3}$ \\ ${ }^{1}$ Universidad de Granada \\ ${ }^{2}$ Ministerio del Interior - Secretaria General de Instituciones Penitenciarias \\ ${ }^{3}$ Universidad Complutense de Madrid \\ Recibido el 07 de mayo de 2020. Aceptado el 05 de septiembre de 2020
}

RESUMEN: La investigación tiene por objeto analizar los derechos de las personas con discapacidad en el medio penitenciario español. En particular, nos centramos en los accesos adaptados para personas con discapacidad física, y los programas y acciones para personas con discapacidad: visión, audición, intelectual y físico. Se utilizó métodos cuantitativos y descriptivos, con un proceso de muestreo biepático compuesto por 310 mujeres $(n=310)$ en proceso de semilibertad, de las que se extrae la submuestra de 59 y 46 quienes reconocen tener algún tipo de discapacidad (grado 1 y 2 , respectivamente). Se aplicó cuestionarios semiestructurados, de los que se esgrimieron preguntas relacionadas con la temática, empleando la base de datos SPSS y Excel, así como estadísticos descriptivos y contraste sobre la asociación (Test Chi-cuadrado) como métodos de análisis de la información. Las 59 mujeres representan el $19 \%$ que tienen reconocida una discapacidad, siendo la física la más recurrente (62.7\%). Los resultados confirmarían que los accesos adaptados con un $45.8 \%$ y los programas y acciones para personas con discapacidad con un $38.4 \%$ son los derechos que menos se cumplen, visibilizando una lamentable realidad en este contexto y que urge la necesidad de mejorar e implementar la protección de derechos de esta población vulnerable.

Palabras clave: prisión; reclusas; discapacidad; derechos humanos; intervención penitenciaria.

Penitentiary environment and the protection of human rights of persons with disabilities. Perspective from the women

\begin{abstract}
The research aims to analyse the rights of people with disabilities in the Spanish prison environment. In particular, we focus on adapted access for people with physical disabilities, and programs and actions for people with disabilities: vision, hearing, intellectual and physical. Quantitative and descriptive methods were used, with a bi-empathic sampling process, consisting of 310 women $(n=310)$, in the process of semi-freedom, from which the subsample of 59 and 46 was extracted, who recognized having some type of disability (grade 1 and 2, respectively). Semi-structured questionnaires were used, using the SPSS and Excel database, as well as descriptive statistics and contrast on association (Chi-square test) as methods of information analysis. The 59 women represent $19 \%$ who have a recognized disability, with physical being the most recurrent $(62.7 \%)$. The results would confirm that adapted accesses with $45.8 \%$ and programmes and actions for people with disabilities with $38.4 \%$, are the rights that are least fulfilled, making visible an unfortunate reality in this context and that the need to improve is urgent and implement the protection of the rights of this vulnerable population.
\end{abstract}

Keywords: prison; inmates; disability; human rights; prison intervention.

Correspondencia: Fernando Gil Cantero. Facultad de Educación. Centro de Formación del Profesorado. Ciudad Universitaria. 28040 Madrid. E-mail: gcantero@edu.ucm.es 


\section{Introducción}

La Declaración Universal de Derechos Humanos (DUDH, 1948) en su artículo 2 señala que toda persona independiente de su raza, color, sexo, idioma, religión o de cualquier otra índole u otra condición, tiene derechos y libertades reconocidos en el ámbito universal (Añaños, 2016). Para poder dar cumplimiento a estos derechos dentro de la prisión, la Organización de Naciones Unidas (ONU) aprobó las Reglas Mínimas para el tratamiento de las reclusas, siendo especialmente aplicables para los propósitos de esta investigación la N. ${ }^{o} 12$ de Bangkok, al señalar que:

se pondrán a disposición de las reclusas con necesidades de atención de salud mental, en la prisión o fuera de ella, programas amplios de atención de salud y rehabilitación individualizados, sensibles a las cuestiones de género y habilitados para el tratamiento de los traumas. (ONU, 2010, p. 12).

En este sentido, constituye también un gran avance la aprobación de La Convención de Naciones Unidas sobre los Derechos de las Personas con Discapacidad (CDPD) (ONU, 2006), al establecer en el artículo 14.2, que:

las personas con discapacidad que se vean privadas de su libertad en razón de un proceso tengan, en igualdad de condiciones con las demás, derecho a garantías de conformidad con el derecho internacional de los derechos humanos y a ser tratadas de conformidad con los objetivos y principios de la presente Convención, incluida la realización de ajustes razonables. (ONU, 2006, p. 10).

En nuestro contexto, el artículo 49 de la Constitución (CE, 1978), fundamenta la intervención especial con internos e internas con discapacidad al establecer la obligación de las Administraciones a promover políticas de "previsión, tratamiento, rehabilitación e integración de los disminuidos físicos, sensoriales y psíquicos" (art. 49 CE). En la misma línea, el artículo 16 de la Ley General Penitenciaria (LOGP, 1979), regula que, con independencia del centro donde ingresen, "los que presenten enfermedad o deficiencias físicas o mentales estarán separados de los que puedan seguir el régimen normal del establecimiento" (art. 16 LOGP, de DIA de MES). En 2018, la Secretaría General de Instituciones Penitenciarias (SGIP, 2018) adoptó el Protocolo de actuación para personas con discapacidad en el medio penitenciario, a fin de identificarlos y proporcionarles un trato adecuado conforme a las necesidades que presenta por su discapacidad. Dentro de este marco legislativo cabe mencionar, por último, el Real Decreto Legislativo 1/2013, de 29 de noviembre, por el que se aprueba el Texto Refundido de la Ley General de derechos de las personas con discapacidad y de su inclusión social (Ministerio de Sanidad, 2013; Paz-Maldonado, 2020).

A pesar de esta protección jurídica y para situar correctamente alguno de los resultados de la investigación que vamos a presentar, es interesante saber que, en España, en 2016, la cifra de incidentes registrada relativos a discapacidad asciende a 262 casos y constituyen el $20.60 \%$ del total de delitos de odio (Ministerio del Interior, 2016, p. 48). Discriminación, que se ve acentuada, por la condición de género (Organización Mundial de la Salud [OMS], 2011) al ser especialmente vulnerables al abuso (ONU, 2020), lo que provoca que las mujeres con discapacidad sufren una doble discriminación (Montón, 2019). Si a esta situación particular de las mujeres le añadimos su privación de la libertad, entonces, no es extraño que sean consideradas uno de los grupos más vulnerables con necesidades y requisitos específicos (Añaños y García, 2017).

En el artículo a fin de conseguir el objetivo general y los específicos; por un lado, considera indispensable conocer la distinción entre los tipos y grados de discapacidad, porque es en función de estos que se delimita el trabajo de campo, enfocado exclusivamente en el Grado 1 y 2 de discapacidad de la persona.

Así, según la clasificación establecida en el Real Decreto 1971/1999, de 23 de diciembre, de procedimiento para el reconocimiento, declaración y calificación, del grado de minusvalía: Grado 1. 
Cuando la discapacidad es nula (cuando la persona sufre daños, tiene síntomas, signos o secuelas, pero no justifican dificultad alguna para realizar las actividades diarias, pero se pueden realizar en su totalidad); Grado 2. Cuando la discapacidad es leve (la persona sufre daños, tiene síntomas, signos o secuelas, y se demuestra que existe alguna dificultad para realizar las actividades diarias, pero se pueden realizar en su totalidad); Grado 3: Cuando la discapacidad es moderada (la persona sufre daños, tiene síntomas, signos o secuelas que causan una importante disminución o imposibilidad de la capacidad de la persona para la realización de las actividades diarias. En este caso, no se ve afectada sus actividades diarias de autocuidado); Grado 4. Cuando la discapacidad es grave (la persona sufre daños, tiene síntomas, signos o secuelas que causan una importante disminución o imposibilidad de la capacidad de la persona para la realización de las actividades diarias. En este caso, puede estar afectada alguna de las actividades diarias de autocuidado); Grado 5. Cuando la discapacidad es muy grave (la persona sufre daños, tiene síntomas, signos o secuelas que impiden e imposibilitan la realización de las actividades diarias).

Y, por otro lado, se analiza los resultados de los cuestionarios semiestructurados, planteados a las internas, respecto al cumplimiento de los derechos a los accesos adaptados para las personas con discapacidad física y, los programas y acciones, para las personas con discapacidad en los centros penitenciarios. Pues bien, para situar correctamente esta investigación, de una lista de veintiocho derechos humanos y fundamentales analizados, los derechos de las personas con discapacidad son los más vulnerados. Así un $10.06 \%$ de la población penitenciaria posee una discapacidad (5,041 personas de los que 4,661 son hombres y 380 mujeres) (SGIP, 2020a). Más en concreto, en los Centros Penitenciarios, la población con discapacidad asciende a 4,610 personas y en el régimen de Semilibertad, 431 padecen una discapacidad (357 son hombres y 74 mujeres) (Ver Tabla 1).

\section{Tabla 1}

Tipos de Discapacidad en Instituciones Penitenciarias (Centros Penitenciarios, CIS y Secciones Abiertas) (diciembre de 2019).

\begin{tabular}{ccccccccccccc}
\cline { 2 - 13 } & \multicolumn{2}{c}{ Psíquica } & \multicolumn{2}{c}{ Intelectual } & \multicolumn{2}{c}{ Física } & \multicolumn{2}{c}{ Sensorial } & \multicolumn{2}{c}{$\begin{array}{c}\text { Pluri- } \\
\text { Discapacidad }\end{array}$} & \multicolumn{2}{c}{ Total } \\
\cline { 2 - 14 } & $N$ & $\%$ & $N$ & $\%$ & $N$ & $\%$ & $N$ & $\%$ & $N$ & $\%$ & $N$ & $\%$ \\
\hline $\begin{array}{c}\text { Centros } \\
\text { Penitenciarios }\end{array}$ & 1,746 & 37.87 & 306 & 6.64 & 1,038 & 22.52 & 134 & 2.91 & 1,386 & 30.06 & 4,610 & 91.45 \\
\hline $\begin{array}{c}\text { CIS y } \\
\text { Secciones } \\
\text { Abiertas }\end{array}$ & 103 & 23.89 & 35 & 8.12 & 185 & 42.92 & 23 & 5.34 & 85 & 19.72 & 431 & 8.55 \\
\hline Total & 1,849 & 36.68 & 341 & 6.76 & 1,223 & 24.26 & 157 & 3.11 & 1,471 & 29.18 & 5,041 & 10.06 \\
\hline
\end{tabular}

Fuente: Ministerio de Interior. Secretaría General de Instituciones Penitenciarias (2020a).

Esta investigación se centra en el estudio de la percepción de reclusas que reconocen tener algún tipo de discapacidad, ya sea física, psíquica, intelectual o sensorial, del grado 1 (discapacidad nula) y/o grado 2 (discapacidad leve), con relación al grado de cumplimiento de los derechos de las personas con discapacidad en el medio penitenciario español. Ahora bien, se han elegido estos dos grados 1 y 2 , ya que las personas con un mayor grado de discapacidad, ya sea del grado 3, 4 o 5 (disminución o imposibilidad de realizar las tareas diarias) no cumplen condena en los mismos espacios de reclusión, ya que existen unos módulos específicos o módulos adecuados al perfil de la persona; y, por lo tanto, en 
la investigación, no se ha tenido acceso a estos módulos específicos o la enfermería, ni tampoco se cuenta con muestra perteneciente a grados más elevados de discapacidad.

Los términos 'discapacidad' y 'ajustes razonables' son entendidos, el primero, según la definición de la OMS (2011):

deficiencias, limitaciones de actividad y restricciones para la participación. La discapacidad denota los aspectos negativos de la interacción entre personas con un problema de salud (como parálisis cerebral, síndrome de Down o depresión) y factores personales y ambientales (como actitudes negativas, transporte y edificios públicos inaccesibles, y falta de apoyo social). (OMS, 2011, p. 7).

El segundo, vehicula la perspectiva de las limitaciones de la discapacidad y las consecuentes políticas de protección y cumplimiento de los derechos humanos en este colectivo, de tal modo que, la ONU propone que en todos los casos y contextos (incluido la prisión) se deben realizar las:

modificaciones y adaptaciones necesarias y adecuadas que no impongan una carga desproporcionada o indebida, cuando se requieran en un caso particular, para garantizar a las personas con discapacidad el goce o ejercicio, en igualdad de condiciones con las demás, de todos los derechos humanos y libertades fundamentales (ONU, 2006, art. 2, p. 5).

\section{Metodología}

\section{Participantes}

Un total de 310 mujeres en cumplimento de condena en medio abierto, es decir, de $3^{\text {er }}$ grado y $2^{\text {do }}$ grado según artículo 100.2 del Reglamento Penitenciario, participaron en este estudio. Las participantes eran mujeres con edades comprendidas entre 18 y 74 años $(M=42.19 D T=10.69)$, las cuales habían estado previamente en prisión. Pertenecían a un total de 31 centros de 13 provincias españolas (Andalucía, Aragón, Castilla y León, Principado de Asturias, Islas Baleares, Canarias, Cataluña, Comunidad Valenciana, Extremadura, Galicia, Comunidad de Madrid, Región de Murcia, País Vasco).

Se crearon dos grupos en función de si las participantes manifestaban alguna discapacidad. El primer grupo estuvo compuesto por un total de 59 mujeres presentaron una discapacidad de grado 1 (discapacidad nula), mientras que el segundo estuvo formado por un total de 49 mujeres con discapacidad de grado 2 (discapacidad leve).

\section{Procedimiento}

La selección de las participantes se realizó durante los meses de junio de 2018 a marzo de 2019. Se siguió como procedimiento un muestreo biepático en dos fases: en la primera fase se seleccionaron los centros por representación territorial y ratio de mujeres. Una vez seleccionados los centros se pidieron los permisos respectivos a las administraciones territoriales y a cada centro. Cuando se obtuvieron dichos permisos y consentimientos, se realizó la segunda fase en la que se llevó al acabo la selección al azar de las participantes del total de mujeres reincidentes de cada centro. A continuación, se informó a las participantes de los objetivos del estudio, de su carácter anónimo y voluntario, así como de su posibilidad de abandonar el estudio en el momento que estimaran conveniente. Una vez su consentimiento informado y firmaron de aceptación voluntaria de participación en la investigación se procedió a la recogida de los datos. Durante el proceso de recogida de los datos estuvo presente un investigador del presente estudio. La aplicación de los cuestionarios fue a pequeños grupos o de manera individual a modo de entrevistas escritas con aquellas que presentaron dificultades de comprensión o de lecto-escritura. El estudio ha sido 
avalado y aprobado por la Secretaría General de Instituciones Penitenciarias, la Consejería de Justicia de la Generalitat de Cataluña, y del Comité Ético de la SGIP.

\section{Instrumentos}

Cuestionarios mixtos diseñados ad hoc. El cuestionario está compuesto de un total de 115 preguntas (sin incluir las entrevistas) organizadas en 6 bloques, que intercalaban preguntas cerradas, múltiples y abiertas. Bloque I: Datos sociodemográficos y jurídicos; Bloque II: Aspectos socioeconómicos e inserción social; Bloque III: Educación y programas; Bloque IV: Prisionización y relaciones de apoyo; Bloque V: Salud y drogas; Bloque VI: Violencia de género y conflictos.

Para el presente estudio, las preguntas utilizadas pertenecen al Bloque V: Salud y drogas, la cual está constituida por un total de 25 preguntas, cuyas temáticas están relacionadas con la salud, drogas, tratamiento de drogodependencias y derechos. En particular, las preguntas seleccionadas del instrumento correspondientes a este bloque son la 82, 82.1 y 100. Las cuales hacen alusión a si tienen o no alguna discapacidad (pregunta 82) y a qué tipo de discapacidad (pregunta 82.1) y, por último, si se cumplen o no algunos de los derechos de las internas en el contexto penitenciario (pregunta 100).

\section{Análisis de datos}

El método de análisis del presente estudio es cuantitativo de tipo descriptivo. Los descriptivo fueron obtenidos usando la Base de datos SPSS versión 24 y Excel. Contraste sobre la asociación entre variables (mediante el Test Chi-cuadrado). Y, análisis de contenido de respuestas abiertas. El margen de error de la muestra es de 4.5 puntos. Con un nivel del $95 \%$ de confianza.

\section{Resultados}

\section{Los derechos de las personas con discapacidad (tipos y grados)}

Conforme a los datos recabados en la encuesta, de las de 310 mujeres, un 19\% ( $n=59)$ tiene reconocida una discapacidad, de las cuales un $15.8 \%(n=49)$ señalan el tipo y grado de discapacidad y, un 3.2\% (n=10) que no responde, y se considera que sí tienen una discapacidad, pero no saben el tipo ni grado (si es discapacidad nula o leve, y el tipo psíquico, intelectual o sensorial), en contrario al $81 \%$ $(n=251)$ que no.

De los datos se extraen que, en primer lugar, las 59 mujeres tienen un tipo de discapacidad de grado 1 (discapacidad nula). El tipo de discapacidad más recurrente es la física con un 62.7\% $(n=37)$, le sigue la psíquica con un $11.9 \%(n=7)$, después la sensorial con un $6.8 \%(n=4)$, la intelectual con un $1.7 \%(n=1)$ y, por último, no respondieron $16.9 \%(n=10)$. Y, en segundo lugar, las 46 mujeres reconocen tener un grado 2 (discapacidad leve) de discapacidad psíquica que representa un $17.4 \%(n=8)$, le sigue la sensorial con un $4.3 \%(n=2)$, la física con $2.2 \%(n=1)$ y, por último, no respondieron $76.1 \%(n=35)$. En este caso, ninguna participante presentó una discapacidad intelectual.

\section{Cumplimiento de los derechos de las personas con discapacidad con relación a los accesos adaptados y a los programas y acciones en los centros penitenciarios}

Conforme a los datos recabados de la encuesta a las reclusas en el medio penitenciario, en cuanto al cumplimiento de los veintiocho derechos humanos y fundamentales, se encuentra que, los derechos de las personas con discapacidad son los derechos que menos se cumplen en comparación con el resto de los derechos. 
Así, 142 internas, de las cuales 49 tienen discapacidad, que representan un $45.8 \%$ del total de respuestas, señalaron que no se cumple el derecho de disponer de accesos adaptados para personas con discapacidad; y 117 internas, un $37.7 \%$, que tampoco se cumple el derecho de participar en programas y acciones para personas con discapacidad.

Acorde a los resultados obtenidos entre el cruce de las variables "Tienes reconocida una discapacidad" y "El cumplimiento de los derechos en el contexto penitenciario", con relación a si hay o no accesos adaptados para personas con discapacidad física, se observó que el $58.1 \%(n=25)$ de las personas con discapacidad reconocían que "No disponían de este tipo de accesos"; mientras que el 27.9\% ( $n=12)$ de personas con discapacidad, indicaban que "Sí, tenían accesos adaptados para personas con discapacidad". Un 14\% $(n=6)$ de las personas con discapacidad dijeron "No Saber si había o no". Una tendencia similar siguió las respuestas de las mujeres que no tenían discapacidad. De éstas, un $50.2 \%$ ( $n=117)$ indicaron que "No había estos accesos", un 33.9\% $(n=79)$ que "Sî", y un 15.9\% $(n=37)$ "No lo sabía". Se realizó una prueba Chi-cuadrado de Pearson para comprobar si existía o no relación entre las dos variables, dicha prueba indicó que no existía esta relación puesto que el p-valor obtenido fue superior a .05 .

Por último, de igual forma que en el caso anterior, se llevó a cabo un cruce entre la variable "Tienes reconocida una discapacidad" y "El cumplimiento de los derechos en el contexto penitenciario", con relación a si hay o no programas y acciones para personas con discapacidad. De acuerdo con los resultados obtenidos, el 51.2\% $(n=22)$ de las personas con discapacidad reconocían que No disponían de este tipo de programas y acciones, mientras que el $23.3 \%(n=10)$ de personas con discapacidad indicaban que Sí tenían programas y acciones para personas con discapacidad. Un $25.6 \%(n=11)$ de las personas con discapacidad dijeron "No Saber, si había o no". Las respuestas de las mujeres que no tenían discapacidad siguieron una tendencia similar al cruce anterior. De estas mujeres, un $41.5 \%(n=95)$ indicaron que "No había estos programas y acciones", un $21.8 \%(n=50)$ que "Sín" y un $36.7 \%(n=84)$ "No lo Sabía". De nuevo, la prueba Chi-cuadrado de Pearson indicó que no existía esta relación puesto que el p-valor obtenido fue superior a .05.

\section{Discusión}

\section{Los derechos de las personas con discapacidad}

Primero. Según los datos recabados en esta investigación el $15.8 \%$ de reclusas, de las 310 mujeres entrevistadas, tiene algún tipo de discapacidad (física, psíquica, intelectual o sensorial) del grado 1 (discapacidad nula) o grado 2 (discapacidad leve). Esto supone casi el doble, si lo comparamos con la población general en régimen de semilibertad, según las cifras proporcionadas por las autoridades penitenciarias, así lo refleja la Tabla 1.

Segundo. Respecto al tipo de discapacidad, en el régimen de semilibertad, la discapacidad física es mayor con relación a las otras discapacidades con un $42.92 \%(n=185)$ del total de personas con discapacidad; le sigue la psíquica, la multidiscapacidad (más de una discapacidad) y, por último, la intelectual y la sensorial. Mientras que, en el régimen ordinario, la psíquica es la que tiene un mayor porcentaje con un $37.87 \%(n=1,746)$ del total, le sigue la multidiscapacidad, la física, la intelectual y la sensorial, sucesivamente. Estos datos difieren levemente en comparación con el estudio: aquí la discapacidad física es la que presenta un mayor porcentaje de casos, con un $62.7 \%(n=37)$, le sigue la psíquica, la sensorial y la intelectual.

Por lo tanto, de los datos se puede interpretar que de los tipos de discapacidad: a) La discapacidad física en el régimen de semilibertad en el estudio de campo tienen los porcentajes más altos en comparación con las otras discapacidades y, en el régimen ordinario, va detrás con un $22.52 \%$ ( $n=$ $1,038)$, de la multidiscapacidad que tiene un 30.06\% $(n=1,386)$, ocupando el tercer lugar; b) La 
discapacidad psíquica (derivada de una enfermedad mental) en el régimen ordinario, tiene el porcentaje más alto, un $37.87 \%(n=1,746)$ con relación al resto de discapacidades. No obstante, tanto en el régimen de semilibertad y en el estudio de campo, está en segundo lugar; esto significa que su incidencia es muy alta. Y, c) La discapacidad intelectual en el régimen de semilibertad tiene un $8.12 \%(n=35)$, casi el mismo porcentaje que la sensorial, que tiene un 5.34\% $(n=23)$. En el régimen ordinario, la intelectual ocupa el tercer lugar con un 6.64\% $(n=306)$, y el cuarto la sensorial con un $2.91 \%(n=134)$; en cambio, en la investigación es la inversa, la intelectual está en último lugar, en consecuencia, hay pocos casos.

Para situar adecuadamente estos datos en la discusión hay que tener en cuenta que, tal vez, las necesidades del cuidado de la salud mental en prisión han de acentuarse porque como advierte el Comité Español de Representantes de Personas con Discapacidad (CERMI, 2019), la discapacidad intelectual y psicosocial suelen pasar, en muchas ocasiones, desapercibidas, frente a las físicas y psíquicas. De hecho, el mismo Defensor del Pueblo (2019a y b) ha señalado que esto puede provocar un "trato inadecuado" y aun alguna "sanción disciplinaria porque se hayan empleado las mismas pautas o criterios que con los demás internos" (Defensor del Pueblo, 2019b, p. 144).

\section{Cumplimiento de los derechos de las personas con discapacidad con relación a la falta de accesos adaptados y programas y acciones en los centros penitenciarios}

Los derechos de las personas con discapacidad están amparados y protegidos en el derecho nacional, supranacional e internacional, así como por parte de diferentes organismos que colaboran en tratar de suprimir o paliar sus obstáculos escolares, sociales y laborales (OMS, 2011). Para nuestra área de interés se ha estipulado así hace tiempo que se "deberán adoptar medidas de protección y promoción de los derechos de los reclusos con necesidades especiales, y dichas medidas no se considerarán discriminatorias" (ONU, 1957, Regla no 2, p. 9).

En concreto, con respecto a la accesibilidad, la Regla N. 5 de Nelson Mandela, señala que los Estados deben facilitar "todas las instalaciones y acondicionamientos razonables, para asegurar que los reclusos con discapacidades físicas, mentales o de otra índole participen en condiciones equitativas y de forma plena y efectiva en la vida de prisión" (ONU, 1957). En España, el acceso de instalaciones para personas físicas ha sido contemplado por una Comisión de estudio sobre los internos con discapacidades intelectuales, físicas y sensoriales, y refieren que las "instalaciones consisten fundamentalmente en la supresión de las barreras arquitectónicas o en la reducción de éstas". En consecuencia y conforme a cada establecimiento penitenciario, se deben realizar las siguientes acciones:

Instalación de rampas en accesos a edificios, patios y zonas comunes; Instalación de barandillas; Habilitación de alguna celda en planta baja en al menos un departamento de los establecimientos o, si las condiciones del edificio lo permiten, instalación eventualmente de ascensores o montacargas e Instalación, en el área donde puedan ser destinadas personas con discapacidad, de servicios higiénicos accesibles o asistidos (MI, 2006, p. 29).

Con respecto a los programas y acciones de tratamiento dirigidas a las personas con discapacidad, cabe considerar el Protocolo de atención a las personas con discapacidad física, sensorial e intelectual (SGIP, 2018) que les permite adaptarse al medio penitenciario según las necesidades educativas de cada tipo de discapacidad. Protocolo que ayuda a la detección/identificación temprana del caso (asignación de módulos sin limitaciones arquitectónicas y la tramitación de certificados), la evaluación, orientación y asesoramiento; la intervención (terapéutica, medidas asistenciales, intervenciones sanitarias; y el seguimiento profesional). Así, en el último Informe de 2018, el Ministerio del Interior, recoge las intervenciones realizadas con las diferentes discapacidades (SGIP, 2019a).

A pesar de todos los esfuerzos realizados en favor de las personas con discapacidad en prisión,

que nosotros valoramos muy positivamente, los datos recabados en esta investigación nos muestran que los derechos de las personas con discapacidad son los que menos se cumplen de todos. En concreto, con 
respecto a la accesibilidad se alcanza un $45.8 \%(n=142)$ del total de reclusas entrevistadas y con los programas y acciones para personas con discapacidad un $37.7 \%(n=117)$, del total. En este sentido, los centros penitenciarios deben asumir las propuestas de la Recomendación 2 del CERMI (2019) cuando señala que es necesario:

la inclusión de un precepto específico en la normativa penitenciaria relativo al tratamiento de las personas internas con discapacidad. En tanto se trata de principios y derechos que poseen una aplicación transversal e inciden en todos los aspectos y dimensiones de la relación jurídica penitenciaria y de la actividad penitenciaria se considera conveniente incorporar este precepto específico entre las disposiciones generales (CERMI, 2019, p. 107).

Alcanzar esta meta implica un trabajo de actuación conjunta de diferentes profesionales, así como firmes políticas de inclusión (Cockram, 2000; Schlanger, 2017) que sitúen la atención al desarrollo integral de las mujeres con discapacidad, privadas de libertad, en un imperativo ético y jurídico a la altura de lo que le corresponde a un Estado social, democrático y constitucional de derecho.

\section{Conclusiones}

Según los datos recabados en esta investigación el número de reclusas con algún tipo de discapacidad es casi el doble de las cifras proporcionadas por las autoridades penitenciarias. La discapacidad física en el régimen de semilibertad en el estudio de campo tiene los porcentajes más altos en comparación con las otras discapacidades. La discapacidad intelectual aparece con pocos casos o iguales que la discapacidad sensorial. La discapacidad psíquica (derivada de una enfermedad mental) tanto en el régimen de semilibertad como en el estudio de campo, está en segundo lugar, aunque su incidencia es muy alta al comprobar que tiene, en el régimen ordinario, el porcentaje más alto con relación al resto de discapacidades. Hay que tener en cuenta, para situar correctamente estos datos, que la discapacidad intelectual suele pasar, en muchas ocasiones, desapercibida.

Por otra parte, según los datos recabados en esta investigación, los derechos de las personas con discapacidad son los que menos se cumplen de todos, algo más con respecto a la accesibilidad y menos con los programas y acciones puestos en marcha para personas con discapacidad, según los cuestionarios realizados a las reclusas.

\section{Agradecimientos}

Agradecemos la financiación de este trabajo al Ministerio de Economía, Industria y Competitividad, la Agencia Española de Investigación y FEDER. Agradecemos el aval de la Secretaría General de Instituciones Penitenciarias y la Consejería de Justicia de la Generalitat de Catalunya. Proyecto I+D+I "Procesos de reinserción y acompañamiento a mujeres en semilibertad" (REINAC), Ref. EDU201679322-R.

\section{Agradecimientos}

Esta investigación se enmarca en el Proyecto $\mathrm{I}+\mathrm{D}+\mathrm{I}$ "Procesos de reinserción socioeducativa y acompañamiento a reclusas en semilibertad" -REINAC- (Ref. EDU2016-79322-R), financiado por el Ministerio de Economía y Competitividad, AEI y FEDER, del gobierno español, liderado por la Universidad de Granada, siendo IP Fanny Añaños.

\section{Referencias}

Añaños, K. (2016). Sistema universal de protección de derechos humanos: análisis de los informes del Comité de Derechos Humanos en Latinoamérica. Revista de Paz y Conflictos, 9(1), 261-278. 
Añaños-Bedriñana, F. T. \& García-Vita, M. M. (2017). ¿Desarrollo humano en contextos punitivos? Análisis socioeducativo desde las vulnerabilidades sociales y el género. Revista Criminalidad, 59(2), 109-124.

CERMI. (2019). Colección Convención ONU 23. La adaptación de la normativa Penitenciaria española a la convención internacional sobre los derechos de las personas con discapacidad. Informe propuesta. Ministerio de Sanidad, Consumo y Bienestar Social. Grupo editorial Cinca.

Cockram, J. (2000). Justice or differential treatment?: Adult offenders with an intellectual disability in the criminal justice system. Theses.

Convention on the Rights of Persons with Disabilities, Nueva York (13 de diciembre de 2006), Resolución 61/106 (13 de diciembre de 2006), Serie de Tratados de Naciones Unidas, vol. 2515, pág. 3, disponible en: https://treaties.un.org/Pages/ViewDetails.aspx?src=TREATY\&mtdsg no=IV$15 \&$ chapter $=4 \&$ clang $=$ en

Declaración Universal de los Derechos Humanos, París (10 de diciembre de 1948), Asamblea General, Resolución 217 A (III), (10 de diciembre de 1948), disponible en: https://www.un.org/es/universal-declaration-human-rights/

Defensor del Pueblo (2019a). Las personas con discapacidad en el Informe Anual 2018. Defensor del Pueblo.

Defensor del Pueblo (2019b). Las personas con discapacidad intelectual en prisión. Separata del Informe Anual 2018. Defensor del Pueblo.

Gil Pérez, D. y Vilches, A. (2017). Educación para la sostenibilidad y educación en derechos humanos: dos campos que deben vincularse. Teoría de la Educación. Revista Interuniversitaria, 29(1), 79100. http://dx.doi.org/10.14201/teoredu29179100

Ley Orgánica 1/1979, de 26 de septiembre, General Penitenciaria. Boletín Oficial del Estado, núm. 239, de 05 de octubre de 1979, pp. 1 a 24. https://www.boe.es/buscar/act.php?id=BOE-A-1979-23708

Ministerio del Interior. (2016). Informe sobre incidentes relacionados con los delitos de odio en España. Secretaría de Estado de Seguridad - Gabinete de Coordinación y Estudios.

Ministerio del Interior, Dirección General de Instituciones Penitenciarias. (2006). Comisión de estudio sobre el abordaje de los internos con discapacidades intelectuales, físicas o sensoriales. Programa de intervención. Documentos Penitenciarios, 5.

Montón Lozano, L. (13 de noviembre de 2019). Un mundo sin barreras. Salamanca.

Naciones Unidas, Comité de Derechos Humanos. (1989). Observación General $n^{o}$ 18: No discriminación. Aprobada en el $37^{\circ}$ período de sesiones (10 de noviembre de 1989). https://www.acnur.org/fileadmin/Documentos/BDL/2001/1404.pdf

Naciones Unidas, Departamento de Asuntos Económicos y Sociales. (2020). Algunos datos sobre las personas con discapacidad, ONU.

Organización Mundial de la Salud. (2011). Informe Mundial sobre la Discapacidad. OMS-Banco Mundial.

Paz-Maldonado, E. (2020). Inclusión educativa del alumnado en situación de discapacidad en la educación superior: una revisión sistemática. Teoría de la Educación. Revista Interuniversitaria, 32(1), 123-146. http://dx.doi.org/10.14201/teri.20266

Real Decreto 1971/1999, de 23 de diciembre de 1999, de procedimiento para el reconocimiento, declaración y calificación del grado de minusvalía. Boletín Oficial del Estado, núm. 22, de 26 de enero de 2000, pp. 1 a 145. https://www.boe.es/buscar/pdf/2000/BOE-A-2000-1546consolidado.pdf

Real Decreto 1/2013, de 29 de noviembre de 2013, por el que se aprueba el Texto Refundido de la Ley General de derechos de las personas con discapacidad y de su inclusión social. Boletín Oficial 
del Estado, núm. 289, de 03 de diciembre de 2013, pp. 1 a 44. https://www.boe.es/buscar/act.php?id=BOE-A-2013-12632

Reglas mínimas de las Naciones Unidas para el Tratamiento de los Reclusos (Reglas de Nelson Mandela), adoptadas en el Primer Congreso de las Naciones Unidas sobre Prevención del Delito y Tratamiento del Delincuente en 1955 y aprobadas por el Consejo Económico y Social, Asamblea General, Resoluciones 663 C (XXIV), (31 de julio de 1957), y 2076 (LXII), (13 de mayo de 1977), disponible en: https://www.ohchr.org/sp/professionalinterest/pages/treatmentofprisoners.aspx

Reglas mínimas de las Naciones Unidas sobre las medidas no privativas de la libertad (Reglas de Tokio), Asamblea General, Resolución 45/110 (14 de diciembre de 1990), disponible en: https://www.ohchr.org/SP/ProfessionalInterest/Pages/TokyoRules.aspx\#: :text=3.8\%20Las\%2 0medidas\%20no\%20privativas,ser\%C3\%A1\%20protegida\%20en\%20todo\%20momento.

Reglas de las Naciones Unidas para el tratamiento de las reclusas y medidas no privativas de la libertad para las mujeres delincuente (Reglas de Bangkok), Asamblea General, Resolución 65/229 (16 de marzo de 2011), disponible en: https://www.unodc.org/documents/justice-and-prisonreform/crimeprevention/65 229 Spanish.pdf

Schlanger, M. (2017). Prisoners with disabilities. En E. Luna (Ed.), Reforming criminal justice: punishment, incarceration, and release (pp. 295-323). Academy for Justice.

Secretaria General de Instituciones Penitenciarias. (2018). Protocolo de actuación para personas con discapacidad en el medio penitenciario. SGIP.

Secretaria General de Instituciones Penitenciarias. (2019a). Informe general 2018. SGIP.

Secretaria General de Instituciones Penitenciarias. (2019b). Programa diversidad: por la igualdad de trato y no discriminación y frente a los delitos de odio - 2018. Documentos Penitenciarios, 20.

Secretaria General de Instituciones Penitenciarias. (2020a). Estadísticas penitencias: distribución de la población reclusa por sexo (diciembre de 2019). SGIP.

Secretaria General de Instituciones Penitenciarias. (2020b). Programas específicos de intervención. SGIP.

Taylor, J., \& Morrissey, C. (2012). Integrating treatment for offenders with an intellectual disability and personality disorder. The British Journal of Forensic Practice, 14(4), 302-315. https://doi.org/10.1108/14636641211283101 